\title{
Are too many septal deviations operated on? A retrospective patient's satisfaction questionnaire with 11 years follow-up*
}

\author{
Navid M. Toyserkani and Thomas Frisch \\ Department of Otolaryngology, Head and Neck Surgery, Rigshospitalet and Faculty of Health Sciences, \\ University of Copenhagen, Denmark
}

Rhinology 50: 185-190, 2012

DOI:10.4193/Rhino11.218

*Received for publication:

October 10, 2011

accepted: November 30, 2011

\section{Summary}

Background: Not much is known about long-term satisfaction of septoplasty. The goal of this study was to assess disease specific quality of life outcomes as well as satisfaction at more than 10 years follow-up after septoplasty.

Methods: The study was conducted as a retrospective questionnaire study (based on the NOSE scale) in which a questionnaire was sent to every patient and followed up by a telephone interview conducted in a tertiary otorhinolaryngologic clinic.

Results: Out of 369 patients who underwent septoplasty, 238 accepted to participate of which 222 were eligible. We found a significant reduction in NOSE score from 56.9 before surgery to 31.9 at follow-up. $68.0 \%$ experienced improved nasal breathing and $55.9 \%$ were satisfied with the overall outcome. Predictive analysis showed that only severity of symptoms before surgery was predictive of all final outcome parameters.

Conclusions: Long-term outcome of septoplasty appears to be in line with shorter follow-up. Surprisingly a sizable difference was noted between rate of satisfaction and improvement in nasal breathing. It is important that the surgeon and patient have the same expectations to septoplasty as to avoid any inappropriate disappointment, which is more likely to occur if symptoms are not severe.

Key words: nasal obstruction, nasal septum, treatment outcome, patient satisfaction, quality of life

\section{Introduction}

Surgical and medical treatments for nasal obstruction are a common part of rhinology practice. A common cause is a deviation of the nasal septum, which has a reported prevalence of up to $80 \%$ in the general population ${ }^{(1)}$. Nasal obstruction can also be caused by other conditions, such as turbinate hypertrophy, adenoid hypertrophy, mucosal congestion, and nasal polyposis. The definitive treatment for a deviated nasal septum is surgical correction, and septoplasty is one of the most commonly performed surgical procedures by otolaryngologists. Nasal airway blockage due to septal deviation may present in various degrees of severity and operation is indicated to improve quality of life through alleviation of symptoms (mouth breathing, snoring, apnea, rhinosinusitis, etc.). However, the long-term outcome of this procedure and its impact on the quality of life has not been clearly established ${ }^{(2)}$.

Outcome after septoplasty may be assessed by objective and subjective measures. Many methods of objective nasal airway evaluation have been proposed including rhinomanometry, acoustic rhinometry, and nasal peak flow. These methods have produced mixed results regarding prediction of outcome after septoplasty and there is no agreement on a measurement tool ${ }^{(3-5)}$.

There have been several prior studies of patients' outcome after septoplasty. Most studies using objective and subjective outcomes have supported that septoplasty is effective in achieving 
the outcomes measured, but most studies, both retrospective and prospective, have had a short follow up of an average of 6 36 months ${ }^{(2,6-13)}$. One study with a follow up of 9 years showed a reduction in the percentage of symptom free patients over time (14). Studies using general health questionnaires have failed to demonstrate changes in patients' quality of life after nasal septal surgery and are not very useful to assess patient benefit ${ }^{(2,6)}$. A disease specific quality of life instrument has been developed for use in nasal obstruction (NOSE scale) for use in prospective studies ${ }^{(15)}$.

The aim of this study was to measure patient satisfaction and symptoms of nasal obstruction using an adapted NOSE scale 11 years after nasal septal surgery with or without additional nasal surgery in a university hospital setting.

\section{Materials and methods}

Setting

This study was performed in a university hospital setting (secondary and tertiary referral center).

Inclusion criteria

Patients who underwent septoplasty in the period of January 1999 and December 2000 were all included in this retrospective study when the indication was nasal obstruction and/or snoring. This included patients undergoing septoplasty alone or in combination with other nasal surgery to represent a typical population representing with nasal obstruction.

\section{Exclusion criteria}

Patients whose indication for septoplasty was not nasal obstruc tion or snoring were excluded. Patients with sinus surgery either before or after septoplasty and patients with acute nasal trauma were excluded from the study. Patients who in the follow-up period had broken their nose were also excluded.

\section{Study design}

The study was conducted as a retrospective questionnaire survey.

\section{Outcome measures}

In spring 2011, all patients were sent a postal questionnaire and asked about their main indications for surgery. The patients were asked about symptoms of nasal obstruction before surgery, using an edited NOSE scale, which used a 5 point scale from 0-4 ${ }^{(15)}$. The NOSE scale has been validated for specific use in patients with nasal obstruction in prospective studies why certain alterations were needed to fit the cross-sectional approach of this study. The NOSE scale includes 5 questions but our survey was supplied with a couple of extra questions previously used in other studies. The patients were also asked about various measures of their satisfaction, and they also had to grade their change in nasal airway breathing and state how pleased they were with the overall final result on a likert scale from 0-4 (Figure 1).

\section{Statistical analysis}

Nonparametric analysis (Wilcoxon signed rank test) was used to compare retrospectively acquired baseline and present follow-up NOSE scores. For two unpaired groups of data, the Mann-Whitney $U$ test was used. Linear regression using forward entry of variables after confirming normal distribution of the dependent variable was used to evaluate predictive factors of improvement in NOSE score. The nonparametric spearman correlation coefficient was used to evaluate bivariate associations. A two-tailed $p$ value less than 0.05 was considered statistically significant.

\section{Results}

A total of 506 patients underwent nasal septal surgery alone or in combination with other nasal procedures over a 24-month period between January 1999 and December 2000. Fifty-seven case notes were missing, and a review of the remaining case notes identified that 9 had concomitant sinus surgery and 5 had acute nasal trauma. Of the 435 remaining patients, 21 were dead, 32 had emigrated and 13 had no known address. Therefore, a postal questionnaire and information letter was sent to 369 patients, which stated that they were going to be contacted by telephone in the near future. We were able to contact 243 of the 369 (66\%) patients of which 5 declined to participate. Sixteen patients were excluded after the interview: 9 patients indicated that their main indication for surgery was other than nasal obstruction or snoring, while 7 had broken their nose after surgery.

In total, there were 222 of 243 (91\%) patients available for analysis, 157 (71\%) were male and the mean age at surgery was 35 (range 15-64). The mean follow-up time was 11.3 years (range 10.3-12.2). Sixteen (7.2\%) had concomitant rhinoplasty and 7 (3.2\%) had concomitant inferior turbinectomy. Twenty-five (11.3\%) patients had previously had septoplasty and together with 7 patients who had a new septoplasty in the follow-up period, a total of 30 patients had the operation more than once. When comparing those who participated and those who did not, there was a difference in mean age (35.47 vs $31.65 p<0.01)$ and a higher percentage of women participated ( $29 \%$ vs $18 \% \mathrm{p}=0.02)$.

There was a statistically significant improvement in NOSE score at follow up (Table 1). In total, 151 (68.0\%) patients felt that their nasal breathing had improved, whereas 71 (32.0\%) patients felt that it had not improved (including 4 who felt that it had worsened). A total of 124 patients (55.9\%) were either pleased or very pleased with the final outcome of the operation. Sixty patients $(27.0 \%)$ were unsatisfied with the operation and $38(17.1 \%)$ were in between (in total $44.1 \%$ not pleased). Twenty-seven (12.2\% of 
Figure 1. Items on the NOSE scale and items using a likert scale.

\begin{tabular}{|c|c|c|c|c|c|}
\hline & Not a problem & Very mild problem & Moderate problem & Fairly bad problem & Severe problem \\
\hline 1. Nasal obstruction & 0 & 1 & 2 & 3 & 4 \\
\hline 2. Nasal congestion & 0 & 1 & 2 & 3 & 4 \\
\hline $\begin{array}{l}\text { 3. Trouble breathing through } \\
\text { nose }\end{array}$ & 0 & 1 & 2 & 3 & 4 \\
\hline 4. Trouble sleeping & 0 & 1 & 2 & 3 & 4 \\
\hline $\begin{array}{l}\text { 5. Unable to get enough air } \\
\text { through my nose during } \\
\text { exercise }\end{array}$ & 0 & 1 & 2 & 3 & 4 \\
\hline 6. Mouth dryness & 0 & 1 & 2 & 3 & 4 \\
\hline 7. Epistaxis & 0 & 1 & 2 & 3 & 4 \\
\hline 8. Anosmia & 0 & 1 & 2 & 3 & 4 \\
\hline \multicolumn{6}{|c|}{ What difference has this surgery done to your ability to breathe through your nose, where 0 is much worse and 4 is much better? } \\
\hline & 0 & 1 & 2 & 3 & 4 \\
\hline \multicolumn{6}{|c|}{ How satisfied are you with the overall final result of your surgery, where 0 is very unsatisfied and 4 is very pleased? } \\
\hline & 0 & 1 & 2 & 3 & 4 \\
\hline
\end{tabular}

How much of a problem were the following conditions for you? Before surgery and now? NOSE score calculations were based on the 5 first questions.

the entire group) patients who indicated that they had improved nasal breathing were not satisfied with the result (Figure 2).

Evaluation of predictive factors showed that the only independent predictive factor for improvement in NOSE score was pretreatment NOSE score, especially sub score 'nasal obstruction' $(p<0.001)$. The pre-surgery NOSE score, however, was not correlated with the overall satisfaction rate. Only the severity of sub score 'nasal obstruction' was correlated with a subjective feeling of nasal breathing improvement $(p<0.001)$ as well as overall satisfaction ( $p=0.028$ ) (Table 2 ). A meaningful cut-off value for preoperative NOSE score was not possible to find using Retriever Operating Characteristics curve.

When looking at the 7 patients who had a new septoplasty in the follow-up period $5(71.4 \%)$ had subjective improvement in nasal breathing and were pleased with the outcome, which was not statistically significant when compared to the remaining sample. In total, 30 patients had more than one septoplasty and when compared to patients with only one surgery, no statistical difference was found. However, a trend towards higher pre- and post-surgery NOSE score was seen in patients with more than one surgery. Likewise, no difference was observed in overall satisfaction rate or subjective improvement in nasal breathing.

There was a significant difference in overall satisfaction rate between smokers who had quit at follow-up and continuing smokers with a higher rate of satisfaction seen in the group who had quit (Table 3). Smoking status at follow-up was correlated with less overall satisfaction ( $p=0.037$ ) but was neither correlated with a lower rate of improvement in nasal breathing, nor with significant changes in NOSE score. There was no correlation with previous nasal trauma or allergic rhinitis and any outcome parameter. Likewise, no demographic (age, gender) or treatment related (rhinoplasty, turbinectomy, same-day surgery vs. inpatient, antibiotics administered) variable was associated with the outcome.

\section{Discussion}

An ideal tool for evaluation of septoplasty does not exist. Outcome research is becoming a significant factor in analysis of clinical practice, and patients' selection often relies on clinical judgment alone. Physical examination and various quantitative measures of the nasal airway do not necessarily correlate with symptoms ${ }^{(16)}$. Because septoplasty is a procedure generally performed to improve nasal symptoms, a nasal-specific questionnaire provides a reasonable approach to the determination of outcome. There have been both retrospective as well as prospective survey studies and the general success rate has been reported to be between $65-89 \%^{(2,3,7,10-13)}$. However, these studies have all had a short follow up (6 - 42 months) compared to this study.

Of the current patients, $68.0 \%$ experienced improved nasal breathing and the overall satisfaction rate was $55.9 \%$. A patient's poor rating of a surgical result may indicate poor 
Figure 2. Overall satisfaction after septoplasty.
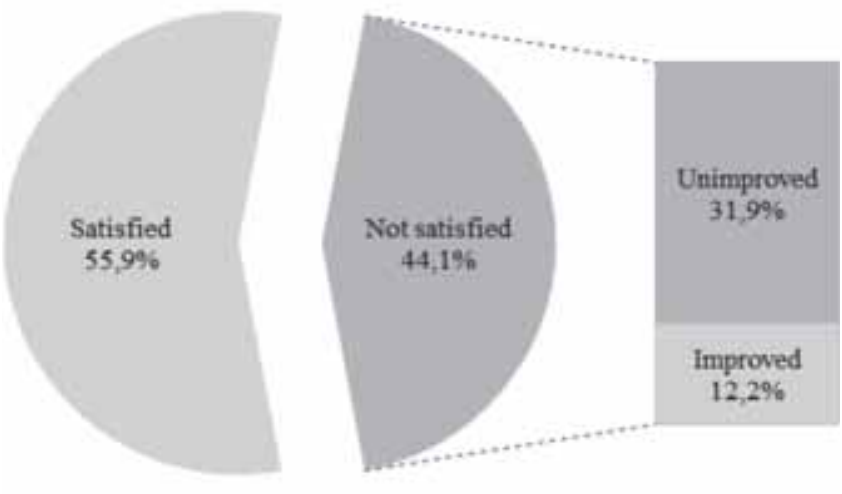

To the left: Satisfied patients have stated to be pleased or very pleased. Not satisfied patient have stated to be very unsatisfied, unsatisfied or in-between. To the right: The not satisfied group is split in two groups based on change in nasal breathing. Of all patients $12.2 \%$ have improved nasal breathing but are not satisfied with the result. surgical technique, inappropriate surgical procedure selection or unrealistic expectations. A study has shown that about $45 \%$ of unsatisfied patients after septoplasty had nasal stenosis caused by septal deviation ${ }^{(17)}$. If true, it would mean that almost half of unsatisfied patients are potentially unsatisfied because of issues directly related to the procedure itself. However, 30 patients underwent more than one septoplasty and there was no difference in the outcome for these patients when compared to the rest, which indicates that the surgical technique was probably not critical to the overall patient dissatisfaction. The problem is, therefore, much more likely to be a combination of inappropriate procedure selection and unrealistic expectations. In this study, it is found that $12 \%$ of all patients experienced improved nasal breathing but were still not satisfied with the outcome. If nothing is gained from a surgical procedure, disappointment is obvious but expectations before surgery should be set so that any improvement in nasal symptoms results in a satisfied patient. On the other hand, some investigators believe that regardless of the severity of septal deviation, most patients benefit from septoplasty because a contributing factor in the pathogenesis of chronic rhinosinusitis is eliminated ${ }^{(18)}$.

Predictor analysis revealed that the severity of symptoms before surgery was predictive of a better outcome after septal surgery. This goes well with commonsense that the worse the symptoms the more potential for improvement. A previous study has shown female gender and previous nasal surgery to be associated with worse outcome, which we were unable to find in this study ${ }^{(2)}$. Smoking status at follow-up was associated with a higher satisfaction rate, but surprisingly, there was no significant difference in NOSE score change or feeling of improved nasal breathing. Smokers who had quit smoking at follow-up had a higher satisfaction rate than continuing smokers. The higher satisfaction rate remains unexplained and as this study was not aimed to test this hypothesis it can only act as pilot data for future studies. A prospective study has shown that patients without allergic rhinitis have a larger improvement after septoplasty; we, however, were not able to find any significant difference between patients with or without allergic rhinitis ${ }^{(8)}$. It should be noted though, that we defined allergic rhinitis only by self-reports and no allergy testing was done as in the prospective study. A randomized study has shown that a better result is achieved if concomitant turbinectomy is done in patients with compensatory inferior turbinate hypertrophy (19). In this study, only 7 patients had the procedure done and could, therefore, not be evaluated properly.

A prospective study has suggested that patients tend to rate the results of their septal surgery increasingly less enthusiastically as time progresses. Also, the initial amelioration of the nasal ventilation felt in the first months or years after surgery is progressively devaluated by the patients with the passing of time, particularly if other causes for nasal obstruction, such as chronic rhinitis and rhinosinusitis, coexist ${ }^{(14)}$. They found a drop in symptom free patients from $51 \%$ at 9 months followup to $26 \%$ at 9 years follow-up. The patients in this study were not asked whether they were symptom free, but in the original NOSE study nonrhinologic patients had a mean NOSE score of approximately $10^{(15)}$. With this definition, $25.7 \%$ of patients were free of symptoms at follow-up in the current study,

Table 1. NOSE scores before surgery and at follow-up.

NOSE Score

Before surgery

56.9 (18.9)

At follow-up 31.9 (23.9)

Values are mean (SD); Higher score indicates worse nasal obstruction. ${ }^{*} p<0.001$ compared with before surgery. 
Table 2. Correlation between Nasal obstruction score before surgery and final outcome parameters.

\section{'Nasal obstruction' score}

Pleased with result
$37.5 \%$

$33.3 \%$

$50.0 \%$

$60.0 \%$

$71.4 \%$

$78.7 \%$
$60.2 \%$

$61.7 \%$

NOSE scores are mean improvement, $(\mathrm{N})$ is number of patients. Percentages are positive outcome based on the likert scales.

Table 3. Smoking cessation and final outcome parameters.

NOSE score before surgery

NOSE score at follow-up

$\%$ with improved nasal breathing

$\%$ who are pleased with result

\section{Stopped smoking}

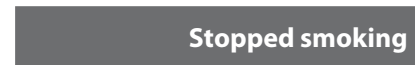

$62.0(21.2)$

$29.8(24.3)$

$72.5 \%$

$70.0 \%$

\section{Still smoking}

60.7 (17.9)

$37.5(25.3)$

$61.3 \%$

$43.5 \%{ }^{*}$

NOSE score values are mean (SD). ${ }^{*} p=0.022$

which is identical to their result. At 9 years of follow-up, 69\% were satisfied with the result, however, their definition of a satisfied patient was one who had improved nasal breathing and the patient's own subjective satisfaction was not evaluated. As such, our rate of improvement in nasal breathing is exactly the same. Another study with 37 patients and a 10 year follow-up had a satisfaction rate of $84 \%$ where patients were selected based on rhinomanometry, in which patients with nasal obstruction due to mucosal swelling were excluded from treatment ${ }^{(20)}$. This indicates that a stricter selection of patients preoperatively may lead to higher satisfaction. It would be optimal if a simple tool as the NOSE score could be used for patient selection, however, no cut-off value could be found in our study; however, our study was not designed to test this. We do not undermine the value of quantitative measures of nasal obstruction, and objective measurements such as Rhinomanometry or Acoustic Rhinometry are possible candidates but still no consensus exists regarding this subject and the focus of this study was on the subjective measures.

Although the current study aimed to evaluate septoplasty outcome in a natural clinical setting, the results of this study should be evaluated with some caution. First, we were only able to contact and include $63 \%$ of eligible patients. It is unclear whether this unwillingness indicates satisfaction or dissatisfaction with the results of the procedure. The participating patients had a slightly higher mean age and we were able to contact a higher proportion of women. These demographic parameters, however, were not associated with any outcome parameter and were therefore not considered to be an important finding. Another weakness of this study is its retrospective design asking patients to remember how their nasal symptoms were 11 years ago. It is difficult to predict in which direction this recall bias has skewed the results of this study but from studies comparing retrospective and prospective assessments, it is known that retrospective assessment in general leads to a slight overestimation of treatment effects ${ }^{(21,22)}$. Still what matters in the end, regardless of 'objective/surgical success', is the subjective satisfaction of the patient, which is not affected in our study.

In conclusion, surgeons should be careful when evaluating patients for septoplasty, as successful straightening of the septum does not necessarily solve the patient's problem. It is important that the surgeon and patient have the same expectations to what can be achieved with a septoplasty as to avoid 
inappropriate disappointment. Patients with worse symptoms tend to have a larger subjective improvement and, therefore, also higher satisfaction rate. Smoking cessation may be beneficial for final satisfaction.

Further studies are needed for the establishment of valid methods for patient selection for septoplasty as to avoid unnecessary disappointment and procedures.

\section{Acknowledgements}

There was no funding for this project.

\section{Authorship contribution}

NMT has gathered all data from case files and telephone interviews, has written the article and done the statistical analysis. TF has supervised the project from planning the project until the end and has contributed to the writing of the article.

\section{Conflict of interest}

There are no conflicts of interest.

\section{References}

1. Gray LP. Deviated nasal septum. Incidence and etiology. Ann Otol Rhinol Laryngol Suppl. 1978; 87(3 Pt 3 Suppl 50): 3-20.

2. Siegel NS, Gliklich RE, Taghizadeh F, Chang Y. Outcomes of septoplasty. Otolaryngol Head Neck Surg. 2000; 122: 228-232.

3. Holmstrom M, Kumlien J. A clinical follow-up of septal surgery with special attention to the value of preoperative rhinomanometric examination in the decision concerning operation. Clin Otolaryngol Allied Sci. 1988; 13: 115-120.

4. Mygind N. Measurement of nasal airway resistance -is it only for article writers? Clin Otolaryngol Allied Sci. 1980; 5: 161-163.

5. Yaniv E, Hadar T, Shvero J, Raveh E. Objective and subjective nasal airflow. Am J Otolaryngol 1997; 18: 29-32.

6. Arunachalam PS, Kitcher E, Gray J, Wilson JA. Nasal septal surgery: evaluation of symptomatic and general health outcomes. Clin Otolaryngol Allied Sci. 2001; 26: 367-730.

7. Gandomi B, Bayat A, Kazemei T. Outcomes of septoplasty in young adults: the Nasal Obstruction Septoplasty Effectiveness study. Am J Otolaryngol. 2010; 31: 189-192.

8. Karatzanis AD, Fragiadakis G, Moshandrea J, Zenk J, Iro H, Velegrakis GA. Septoplasty outcome in patients with and without allergic rhinitis. Rhinology. 2009; 47: 444-449.

9. Konstantinidis I, Triaridis S, Triaridis A, Karagiannidis K, Kontzoglou G. Long term results following nasal septal surgery. Focus on patients' satisfaction. Auris Nasus Larynx.
2005; 32: 369-374.

10. Pirila T, Tikanto J. Unilateral and bilateral effects of nasal septum surgery demonstrated with acoustic rhinometry, rhinomanometry, and subjective assessment. Am J Rhinol. 2001; 15: 127-33.

11. Samad I, Stevens HE, Maloney A. The efficacy of nasal septal surgery. J Otolaryngol. 1992; 21: 88-91.

12. Stewart MG, Smith TL, Weaver EM, et al. Outcomes after nasal septoplasty: results from the Nasal Obstruction Septoplasty Effectiveness (NOSE) study. Otolaryngol Head Neck Surg. 2004; 130: 283-290.

13. Uppal S, Mistry H, Nadig S, Back G, Coatesworth A. Evaluation of patient benefit from nasal septal surgery for nasal obstruction. Auris Nasus Larynx. 2005; 32: 129-137.

14. Jessen M, Ivarsson A, Malm L. Nasal airway resistance and symptoms after functional septoplasty: comparison of findings at 9 months and 9 years. Clin Otolaryngol Allied Sci. 1989; 14: 231-234.

15. Stewart MG, Witsell DL, Smith TL, Weaver EM, Yueh B, Hannley MT. Development and validation of the Nasal Obstruction Symptom Evaluation (NOSE) scale. Otolaryngol Head Neck Surg. 2004; 130: 157-163.

16. Hardcastle PF, White A, Prescott RJ. Clinical or rhinometric assessment of the nasal airway-which is better? Clin Otolaryngol Allied Sci. 1988; 13: 381-385.

17. Dommerby H, Rasmussen OR, Rosborg J. Long-term results of septoplastic operations. ORL J Otorhinolaryngol Relat Spec.
1985; 47: 151-157.

18. Elahi MM, Frenkiel S. Septal deviation and chronic sinus disease. Am J Rhinol. 2000; 14: 175-179.

19. Devseren NO, Ecevit MC, Erdag TK, Ceryan K. A randomized clinical study: outcome of submucous resection of compensatory inferior turbinate during septoplasty. Rhinology. 2011; 49: 53-57.

20. Bohlin L, Dahlqvist A. Nasal airway resistance and complications following functional septoplasty: a ten-year follow-up study. Rhinology. 1994; 32: 195-197.

21. Aseltine RH, Jr., Carlson KJ, Fowler FJ, Jr., Barry MJ. Comparing prospective and retrospective measures of treatment outcomes. Med Care. 1995; 33(4 Suppl): AS67-AS76.

22. Dawson EG, Kanim LE, Sra P, et al. Low back pain recollection versus concurrent accounts: outcomes analysis. Spine (Phila Pa 1976). 2002; 1, 27: 984-993.

Navid M. Toyserkani

Slotsfogedvej 72 .tv

2400 København NV

Denmark

Tel: $+45-50383833$

E-mail: drg777@alumni.ku.dk 